\section{LECTURES

\author{
ON THE
}

RELATIONS OF SARCOMA TO CARCINOMA.

\section{Delivered at the Royal College of Surgeons of England, June I880.}

BY HENRY TRENTHAM BUTLIN, F.R.C.S.,

Erasmus Wilson Professor of Pathology to the College; Assistant-Surgeon to St. Bartholomew's Hospital; etc.

Lecture III (Abstract). - Central Sarcoma of Bone.

To the round-celled, spindle-celled, and mixed-celled groups, in which the subperiosteal tumours were arranged, must now be added a fourth group, to comprise the myeloid or giant-celled sarcomata. In this group I shall include only those tumours which are so largely constituted of giant-cells that these cells produce a decided effect upon the appearance of the tumour-in other words, that they colour the tumour maroon or red.

The 63 cases of central sarcomata here collected include 20 roundcelled, 18 spindle-celled, 9 mixed-celled, and 16 giant-celled. For the better comparison of the central sarcomata, it will be convenient to adopt the same method as was employed in considering the subperiosteal tumours, and to study separately the tumours of the various bones affected. Those of the femur are again more numerous than the tumours of any other bone.

The round-celled tumours occurred in patients, most of whom were older than those who were the subjects of subperiosteal round-celled tumours. Only one case was of the upper epiphysis. In this case, the tumour became widely generalised. Among the organs affected by it were the lymphatic glands-a circumstance so rare, that in every case where it is noted I shall try with care to ascertain the cause. In this instance, it was probably due to the infiltrating nature of the tumour, which spread to the adductor muscles, whence it could obtain easy access to the deeper femoral glands, and thus spread along the lymphatic channels. With the exception of this case, these tumours were less malignant than the corresponding tumours of superficial origin.

One of the spindle-celled tumours, which grew from the upper epiphysis of the femur of a woman, thirty-six years old, was distinguished by being composed chiefly of cartilage of various forms, but for the most part cellular. It had been five or six months growing, when amputation at the hip-joint was performed. The patient died from blood-poisoning within three weeks of the operation, and at the necropsy a secondary nodule was found in the right lung. This case is mentioned particularly, because by many pathologists it would be regarded as one of enchondroma. But it is here ranged among the sarcomata on account of its rapid course and secondary affection of the lungs, and of the rich mingling with the cartilage of spindle-shaped and other cells. Affection of the glands was associated with one spindle-celled tumour, which, like the last, grew in the upper epiphysis. Of the relation which this glandular disease bore to the tumour I am not sure, but imagine the glands were affected through the medium of the blood and not of the lymph; for the femoral glands were not enlarged, those of the groin scarcely at all; while the glands of the pelvis and of one axilla were extensively diseased. If the disease of the pelvic and axillary glands was of the same nature, it is evident both sets of glands could not have been affected through the lymphatics from a single source.

The sub-group of mixed-celled tumours contains little of marked interest; for two of the patients were not traced after their recovery from amputation, and the third patient died from operation.

Of the myeloid tumours, I may point out that all five were unmixed with cartilage or similar tissue, and that neither of them grew into the surrounding soft parts. One case is distinguished by its fatal termination. In some respects, its history is very characteristic, not only of myeloid, but of other central tumours. Thus, the first symptom was pain, which endured a month, when the femur gave way almost spontaneously; but it was not till three or four months after the fracture the presence of a tumour was observed.

Regarding, now, the characters common to most of these tumours of the femur, the most apparent and most important are their less fatal nature, compared with the subperiosteal tumours, and often slower course. Of three patients, it is recorded they were alive and well at periods respectively of nine months, sixteen months, and three years after operation.
Only eight deaths are reported-a mortality strikingly less than that of the cases of subperiosteal disease. Even of these eight deaths, three were due to causes directly connected with operation. Extensive generalisation was very rare. Affection of the glands, too, was very unfrequent. A noticeable feature in this group of tumours is the comparative simplicity of their structure; for, if we exclude two in which bony spicula were present, there remain only two with which cartilage was mixed. A strong disposition of central tumours to attack the lower epiphysis, or lower third, is very apparent. Lastly, many of the patients were older than those who suffered from subperiosteal tumours, the range of age extending from thirteen to fifty-seven, instead of from nine to forty-one; while the distribution of cases among the decades was pretty equal.

With perhaps one exception, the tumours of the tibia bear a similar relation to the tumours of the femur to that which exists between the subperiosteal tumours of the same bones. The exception is the rarity of ulceration of the integument. The points of accordance are the slightly more advanced age of the patients; the position of the growth, generally in the upper epiphysis of the bone; the longer duration of the cases, exhibited in the columns of duration to operation, of total duration, and in the final column; the absence of recurrence in the stump; and of affection of the glands. Let me here draw attention to the resistance offered by the articular cartilage to the growth of central tumours, not only of the tibia, but of all long bones. It maintains its integrity long after the hardest bone has been destroyed.

The two tumours of the fibula may be regarded as a kind of appendix to the tumours of the tibia. They occurred during the middle period of life, were of the upper epiphysis, and both displayed a less malignancy than the corresponding subperiosteal tumours.

The spindle-celled tumour of the foot occurred in the form of isolated medulla-like growths in the interior of several adjacent bones of the tarsus and metatarsus. The most interesting feature of the case was the condition of the lymphatic glands, for one popliteal, the lower inguinal, the pelvic, and the medistinal, were all affected by the disease. This is the only case of central tumour associated with affection of the glands in which the disease appeared to travel through the lymphatic channels directly from the bone. Nor can I offer any explanation of the cause of what appears to be a deviation from the common rule.

The first case of the group of tumours of the humerus is also an example of glandular affection, but of a different kind. There was a tumour of each humerus, and one of the medulla of one femur, but the mesenteric and retroperitoneal glands were the only soft parts affected. The relation of this gland affection to the tumours of the bones is not clear, but the disease could scarcely have been conveyed through the lymphatic channels.

The tumours of the ulna and radius attacked almost always the lower portion of each bone. A very modified malignancy is evident in four of the five cases, for even the fatal case is marked by a complete absence of secondary disease.

The cases of tumour of the scapula would be more numerous, were it not for the difficulty of deciding on their origin.

The central tumours of the lower jaw are far more numerous than those of subperiosteal origin, but I imagine No. 47 would more rightly be classed among the latter. Recurrence after operation is noted only of two of these cases, the one I have just mentioned, and a case in which the tumour was simply enucleated from the cavity in which it lay. In the latter the progress of the recurrent growth was very slow.

The tumour of the sternum might fairly be included with those of the skull ; or might, perhaps, more rightly form one of a group, the members of which attack several bones almost at the same time.

The pelvic tumour did not affect the lymphatic glands, and the reason for this forbearance may probably be found in its relation to the cavity of the pelvis, and, therefore, to the glands. Almost the whole of the sacrum and coccyx was replaced by the new growth, which infiltrated the muscles posteriorly, but was limited in front by the periosteum, which separated it from the interior of the pelvis.

The tumours of the "skull differ from the cranial subperiosteal tumours, first and most strikingly in the much older patients they attacked; second, in the fact that, although three of them were of the vault, only one produced multiple tumours of the skull. As in the subperiosteal tumours, so in two of these, there was wide-spread generalisation, chiefly in various bones. This leads me to speak of a class of cases in which many bones are attacked almost simultaneously by sarcomatous disease. This, I imagine, was the nature of a case recorded by Dr. Moxon* in the Pathological Transactions. Of course it cannot be absolutely asserted that most of these tumours were not secondary to one among their number. But their near equality in size, the absence of symptoms

\footnotetext{
* Patho'ogical Transactions, vol. xxii., page 206 (187r).
} 
Central Tumours of Bone.

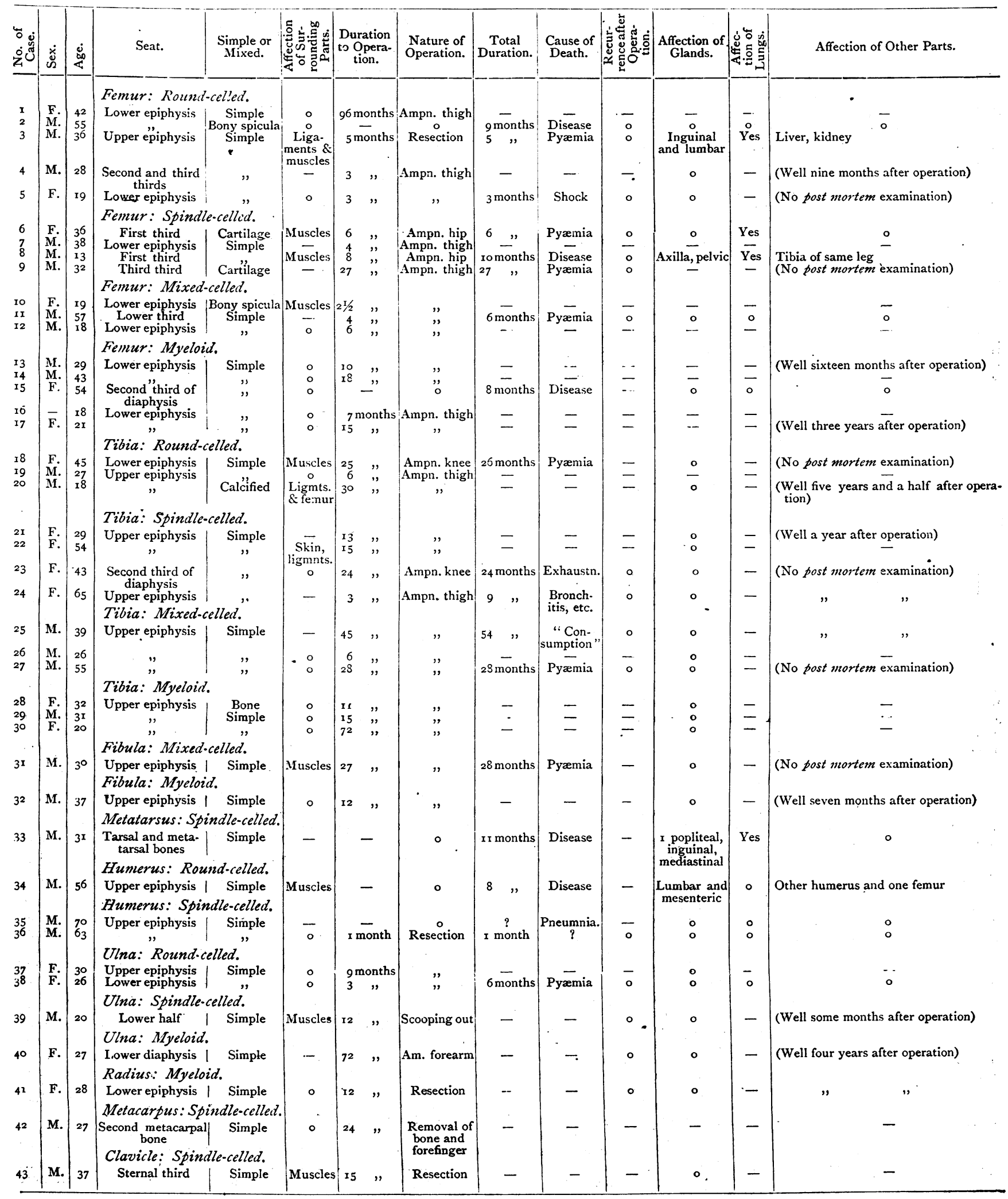


Central Tumours of Bone-continued.

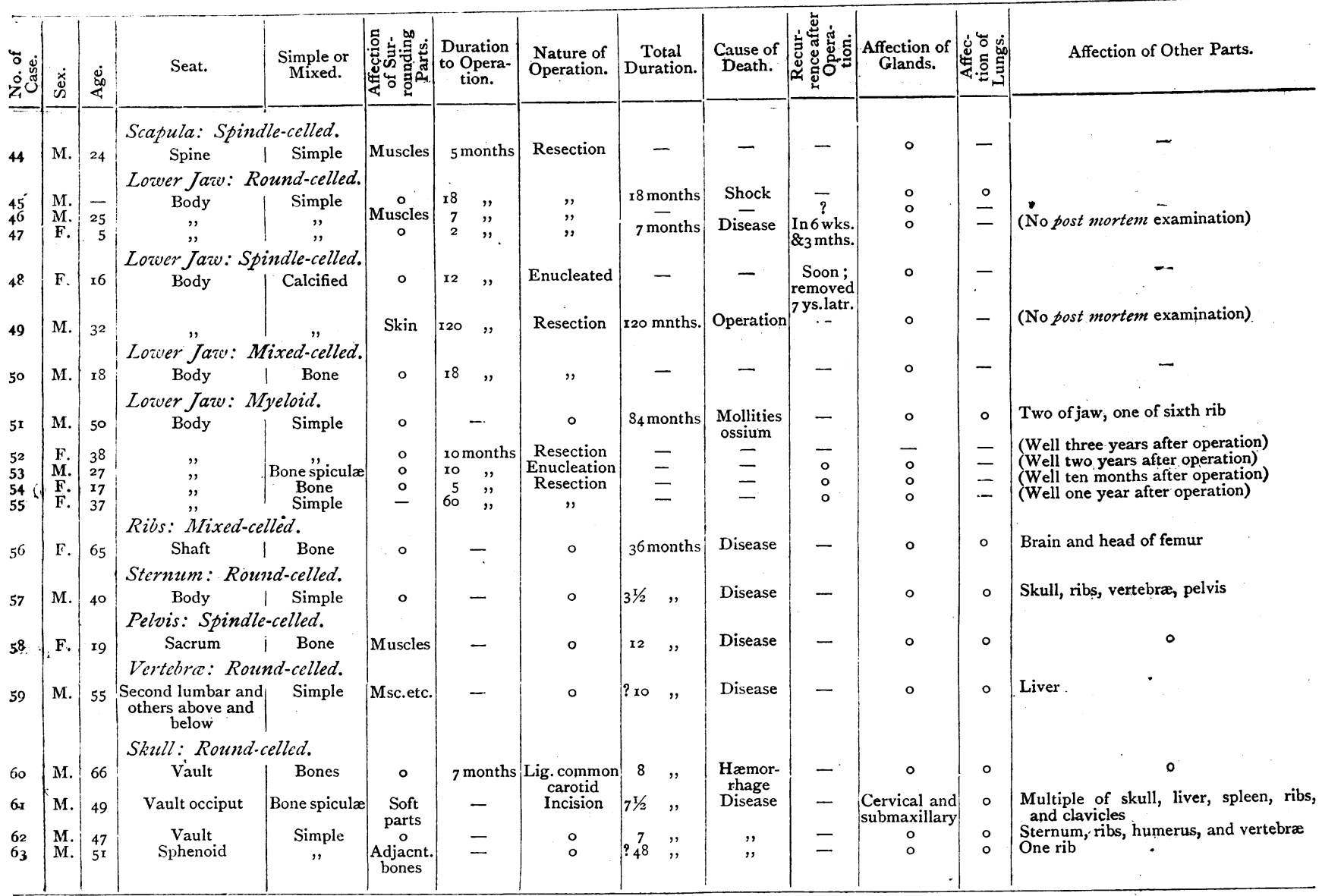

indicating long duration of any of them, and the absence of secondary affection of parts usually prone to such affection, all point to another origin than this. Probably several of the cases in this table are instances of this general sarcomatous disease; for example, the last three of those of the skull, that of the sternum, and perhaps the round-celled tumour of the humerus. All the tumours were round-celled, and the bones most frequently affected were the skull, the ribs, and the vertebræ.

Hitherto I have dwelt chiefly on the characters of the tumours of particular bones. Now let me point out certain features common to either or both of the two large classes. r. As to their history and course : central tumours generally occur in persons older than those who are the subjects of subperiosteal tumours. Central tumours grow more slowly than do subperiosteal. Neither injury nor inflammation appears to exercise much influence in the production of either class of tumour 2. The physical conditions of the tumours previously to their removal or to death are interesting in two particulars, their occasional pulsation and their situation. Pulsation was present more often in central than in subperiosteal tumours, in either case only in the tumours of certain bones, the femur, tibia, calvaria, and innominate bone. Where the cause of the pulsation could be ascertained, it was in every instance due to an abundant supply of vessels of moderate calibre.

Long bones, short bones, and flat bones are liable to sarcoma, but not all bones equally, nor even all the bones of each shape in equal proportion. The long bones of the lower extremity are more frequently attacked than the bones of the arm and forearm, the bones of the metatarsus and metacarpus are rarely affected, the phalanges almost never. The flat bones of the skull, the scapula, and innominate bone, are al subject to sarcoma, but the skull-bones most of all. None of the short bones are very liable to the growth of primary disease. But, more than this, these tumours show a decided preference for the articular ends or epiphyses of the long bones, and especially the tumours of central origin for one articular end of each long bone. In the upper ex- tremity it is the upper epiphysis of the humerus, the lower of the radius and ulna ; in the lower extremity it is the lower epiphysis of the femur, the upper of the tibia and fibula, which are most frequently affected. To these parts the following conditions appear to be common. They contain cancellous tissue in greater or less abundance; their position renders them liable to injuries of various kinds : in the process of development of the bones they are the parts which are the first to ossify, the last to be united with the shaft, and the direction of the nutrient artery is not towards but from them. 3. After removal of the tumour, a radiating appearance is observed on section of most subperiosteal tumours, which probably is solely due to the manner in which the tumour grows or is developed. For the chief growth or development takes place around the tiny vessels which pass from the periosteum almost at right angles through the cortex of the bone; and each of these becomes the centre of a column resting on the bone. 4, Their progress and termination show that all sarcomata of bone may be malignant, but their malignancy is of widely different degrees. Speaking generally, it may be said that central sarcomata are far- less malignant than subperiosteal, and those of bones more distant from the trunk than those of bones more near. The former of these statements inay be illustrated by the cases I have myself observed. Of eleven patients with subperiosteal sarcoma of various bones, I know that nine are dead. The tenth, whose tumour was of the tibia, and for whom amputation was performed at the end of two months, was alive and well when last heard of, fourteen months after the operation. In the eleventh case, amputation was performed scarcely more than a few weeks ago. Of 10 cases of central tumour, I have notes of the death of 3 , of slow recurrence in a fourth case during seven years after operation, and of the good health of 4 patients at periods respectively of $\mathrm{I}, 3 \frac{1}{2}, 4 \mathrm{~m}$ and $51 / 2$ years after operation. The least degree of malignancy is the infiltration of contagious structures, much more common in subperiosteal than in central tumours. Next in order is affection of the lymphatic glands, rarely occurring in connection with subperiosteal and still more 
rarely with central tumours. It may be of the same nature as the affection of other organs where the disease is conveyed through the medium of the blood. Or it may probably originate in continuous infiltration of one or more lymphatic glands by the primary disease. Or, since I am not able to affirm the contrary, the disease may be conveyed through the lymphatic channels directly from the bcne; but I venture to think this is the least common method of invasion, if indeed it ever occur, in comnection with sarcomata of bone. The last degree of malignancy is that in which growths appear in organs and tissues more or less remote from the primary disease, in most cases undoubtedly conveyed thither by the blcod, in some cases apparently due to distinct outbreaks of sarcomatous disease. Of all organs the lungs are far more commonly affected than any other, and the tumours which exhibit the most marked tendency to wide-spread generalisation are the roundcelled tamours. But it is the round-celled tumours of certain bones which are thus liable to become widely generalised, and the subperiosteal which are far more likely than the central tumours to affect the lungs.

With regard to operation, it appears as if, setting aside cases of multiple sarcoma and of tumours so situated that they cannot be removed, no doubt can exist of the propriety of operation in all cases where the disease has not advanced too far. The advantages of operation are obvious. Complete removal of the primary disease arrests its continuous infiltration, and terminates the dangers incidental to the mere presence of a tumour, often of enormous bulk; for local recurrence may be regarded as an accident which might absolutely have been prevented by free removal; an accident almost unknown, when, for example, the thigh is amputated for a tumour of the tibra. Nor does there appear sufficient ground to doubt that, if the primary tumour be early enough removed, generalisation by the blood and affection of the glands may be prevented. Against these benefits the only disadvantage which can fairly be alleged is the danger of the operation itself, a danger marvellously less at the present time than it was even ten years ago, and not to be gauged by the horrible mortality exhibited in this table of central sarcomata.

\section{TYPHOID FEVER IN NEW ZEALAND AND AUSTRALIA; ITS ORIGIN AND PROPAGATION.}

By J. RUTHERFORD RYLEY, F.R.C.S., L.R.C.P.Edin., etc., Formerly Provincial Surgeon of Westland, New Zealand; Surgeon to Provincial Hospital, Gaol, and Lunatic Asylum; Police-Surgeon and Medical Assessor for the Province, etc.

THE conflict of opinion which still exists on the question of the origin and propagation of typhoid fever is, I think, a sufficient justification for this contribution to the literature of the subject from our Australian colonies, where the disease has occurred, and still occurs, under circum. stances which render the solution of the problem less difficult, I think, than has been found to be the case in older countries, such as England, even in rural districts, the favourite habitat of the disease. The publication of the paper may, moreover, and I hope will, stimulate others of the profession in the colonies to publish their experience on a subject in itself of much pucely scientific interest to the medical philosopher, but of still greater interest alike to the practical physician, sanitarian, and legislator. Drs. W. Budd and Murchison are, in this country, the authorities whose views on the subject are representative. Both admit a plurality of poisons in the case of continued fever, and the specificity of the poison of typhoid fever. Dr. Budd is of opinion that the specific poison or contagium of the fever in question is invariably derived from a specific eruption of the intestine, like that of small-pox, which, on being discharged from the bowels with the excreta, constitutes the whole source of the disease. Dr. Murchison, while denying that the fresh dejections from the bowels of the affected person contain any such poison, admits that feculent matter in a state of fermentation or putrefaction, after its discharge from the bowels even of a person not at the time affected with the fever, may produce the contagium, and perhaps is the chief source of the disease; but he asserts that other organic matter especially of an animal nature in a state of fermentative putrefaction, is capable, under certain unknown conditions of the atmosphere, etc. of originating de novo the contagium. Both these distinguished authorities, however, are agreed that the great cause of the disease is the contamination of drinking-water by sewage or excremental matter and both admit that the poison may be conveyed in other.ways, such as by milk, and perhaps by the air-fomites, etc. The one considers the contagium very active and virulent; the other regards it as com paratively mild, and only very slightly contagious.

It is beyond the limits of this paper to enter on the discussion of dis. puted points. I proceed to give, as briefly as possible, my own experience on the subject, and the conclusions I have formed from such experience. Early in. Jankary 1864, I arrived in Westland, New Zealand, at the commencement of the great gold rush which occurred there. Only a few years before, this rich province-a large strip of , land $^{2}$ about two hundred miles in length, by an average breadth of thirty miles; on one side, a high range of mountains, which runs like a bagkbone throughout these islands; on the other, the Pacific Ocean-was almost a terra incognita, having only been visited by Dr. Hector, of the Geological Survey of New Zealand, and a few surveyors, and was inhabited only by a handful of aborigines, who lived on fish and the natural productions of the soil. The land was thickly timbered, and so covered by a dense jungle of scrub and undergrowth as to be almost impenetrable, so that the miner or "prospector". had to clear his. way axe in hand. The rainfall, which in New Zealand generally is about ten inches higher than in England, was then at all events , unusually high in Westland. The miner prospecting or travelling, thesrefore, was almost constantly wet, either from the direct xainfall, or ifrom the drops from the trees or shrubs, or from the rank vegetation that every where covered the ground. Coupled with this, his scanty fare of "damper" of unleavened bread and perhaps bacon, and tea without milk, and his hard work and exposure, being sheltered at night only by his $\mathrm{V}$ tent, what wonder is it that he was attacked with disease? The chief, diseases were fever, dysentery, rheumatism, and inflammatory chest-affections. Many cases of simple fever and a fow of bilious fever occurred in the early days of the "rush", and I saw then a few cases of ague also, but only in those predisposed by previous attacks elsewhere, as in South America. I was appointed by the Government surgeon to the hospital, and for the first three years I had, at an average, twenty cases of fever constantly under treatment there, besides what I saw in private practice. This number gradually diminished until, when I resigned in the end of 1869, there were never more than two or three cases. I have not the figures before me, but I am sure I saw not less than four hundred cases during the time $I$ was there, nearly five years. As is the case in opidemics generally, the disease was much more severe, during the first six months, becoming: gradually milder and the number attacked fewer, as time wore on. As imuch doubt was entertained by the profession , at the time, both in New.Zealand and Australia, not only of the genesis or etiology of the fever prevailing there, but as to its real nature, the name given to it by the public, and pretty generally adopted by the profession being "colonial fever", I resolved, having a large field for observation, to investigate the matter by a careful inquiry into: the history of each case, by carefully recording the symptoms, and by making post mortem examinations of nearly every case of death from fever that occurred in hospital. Suffice it to say that the history, symptoms, course, and complications, together with the intestinal lesions discovered in every case after death, were those of typhoid or enteric fever. Among the preparations left by me at the hospital, showing the characteristic ulcerations of Peyer's glands, there were three where the bowels were completely perforated. The results were given by me in my annual reports published by the Government at the time. The'persons attacked were chiefly the miners, new arrivals prospecting in the bush or working in their claims, and came not from any one part of the district, but from a circuit of fifty miles or more around. Very few indeed, comparatively, came from the townships or centres of population, nearly all being miners or others working in the "bush". I remember no case of relapse having occurred, and I know of no case of a second attack of the fever having occurred in any case.

What, under such circumstances, was the proximate cause of the fever? It could not be contagion, as the cases occurred sporadically all over the district simultaneously or in succession, and without any intercommunication-not from any centre or centres of infection. It coild scarcely be excremental matters in a state of fermentative putrefaction, as many of the earlier cases occurred amongst prospectors shifting their. camps daily or oftener; nor could it, I think, be animal matter in a state of decay. The water everywhere was loaded with vegetable matter in a state of more or less decay. This water was used by the miners as their ordinary beverage, and for making their tea, etc. ; and to this cause, in my reports at the time, I attributed the fever; and my subsequent experience has only sufficed to confirm this opinion, especially my experience in Fiji, vit, that enteric fever may be produced independently of contagion, whtheter thay be its other mode of origin from the contamination of the drinking water, and perhaps, also the air by deoaying organic'matter of a 'vegetable nature. I have seen cases of the fever since in Fiji and Western Australia, both in the tropics and in South Australia, and New South Wales, in some cases apparently from this cause, there being no other cause apparent, certitinly. not contagion; in 'other cases apparently from the contamination of the drinking : water, by human excretal matter, but in not à 